\title{
Combination effect of green tea extract and L-theanine on memory and attention in mild cognitive impaired subjects: a randomized double-blind placebo-controlled study
}

\author{
S. K. Park ${ }^{1}$, I. Jung ${ }^{2}$, W. K. Lee ${ }^{1}$, Y. S. Lee ${ }^{1}$, H. K. Park ${ }^{1}$, H. J. Go ${ }^{3}$, N. G. Yim ${ }^{4}$, J. T. Hong ${ }^{5}$, \\ S. Y. Ly ${ }^{6}$ and S. S. Rho \\ ${ }^{1}$ LG Household and Health Care Co. Ltd, Daejeon, Republic of Korea, ${ }^{2}$ Department of Neuropsychiatry, Daejeon \\ University, Daejeon, Republic Korea, ${ }^{3}$ Department of Psychiatry, Catholic University, Daejeon, Republic of Korea, ${ }^{4}$ Human \\ Ecology Institue, Seoul, Korea, ${ }^{5}$ Department of Pharmacy, Chungbuk National University, Cheongju, Republic Korea, \\ ${ }^{6}$ Department of Food Nutrition, Chungnam National University, Daejeon, Republic of Korea and ${ }^{7}$ Department of \\ Ophthalmology, Otolaryngology and Dermatology, Daejeon University, Cheongju, Republic of Korea
}

The combination of green tea extract and L-theanine (LGNC-07) has been reported to have cognition improving effect in animal studies $^{(1)}$. In this randomized, double-blinded placebo-controlled study, we investigated the effect of LGNC-07 on memory and attention in the mild cognition impaired (MCI) subjects ${ }^{(2)}$. Ninety-one MCI subjects whose MMSE-K scores were between 21 and 26 with $2-3$ stages of GDS were enrolled in this study. The treatment group (13 males, 32 females; $57.58 \pm 9.45$ years) took $1440 \mathrm{mg}$ GTE and $240 \mathrm{mg}$ L-theanine and the placebo group (12 males, 34 females; $56.28 \pm 9.92$ years) took the equivalent amount of maltodextrin and lactose for 16 weeks. Rey-Kim memory test, Stroop colour-word test were conducted to evaluate the effect of LGNC-07 on memory and selective attention.

\begin{tabular}{|c|c|c|c|c|c|c|c|c|}
\hline & \multicolumn{4}{|c|}{ Change at 8 weeks } & \multicolumn{4}{|c|}{ Change at 16 weeks } \\
\hline & \multicolumn{2}{|c|}{ LGNC-07 $(n$ 11) } & \multicolumn{2}{|c|}{ Placebo $(n 9)$} & \multicolumn{2}{|c|}{ LGNC-07 $(n$ 11) } & \multicolumn{2}{|c|}{ Placebo $(n 9)$} \\
\hline & Mean & $\mathrm{SD}$ & Mean & $\mathrm{SD}$ & Mean & $\mathrm{SD}$ & Mean & SD \\
\hline $\begin{array}{l}\text { Rey-Kim memory test } \\
\text { Memory quotient (MQ) } \\
\text { Verbal learning test }\end{array}$ & $8.82^{\mathrm{b}}$ & 10.39 & 4.78 & 10.44 & $14.18^{\mathrm{a}, \mathrm{b}}$ & 10.56 & $6.00^{\mathrm{b}}$ & 7.19 \\
\hline Trial 1 & $1.91^{\mathrm{b}}$ & 2.26 & 1.44 & 2.70 & $3.18^{\mathrm{b}}$ & 1.54 & $3.00^{\mathrm{b}}$ & 2.35 \\
\hline Trial 2 & 0.73 & 2.37 & 0.56 & 1.59 & 1.09 & 2.81 & $1.67^{\mathrm{b}}$ & 1.73 \\
\hline Trial 3 & 1.18 & 2.18 & -0.22 & 2.99 & $1.91^{\mathrm{b}}$ & 2.55 & -0.22 & 2.99 \\
\hline Trial 4 & 0.82 & 1.72 & 0.44 & 1.81 & 1.00 & 2.24 & 0.89 & 1.90 \\
\hline Trial 5 & 0.73 & 2.76 & 1.33 & 2.29 & -0.18 & 1.99 & 0.78 & 2.68 \\
\hline Delayed recall & 1.18 & 2.79 & 0.78 & 3.70 & 2.64 & $3.04^{\mathrm{b}}$ & $1.89^{\mathrm{b}}$ & 2.26 \\
\hline Delayed cognition & 1.45 & 4.03 & 1.73 & 4.05 & 0.89 & 3.59 & 1.33 & 2.40 \\
\hline Stroop color word test & & & & & & & & \\
\hline Word reading & $3.18^{\mathrm{a}}$ & 7.04 & -0.44 & 1.88 & 1.91 & 6.27 & 0.11 & 2.57 \\
\hline Color reading & 8.27 & 20.53 & 0.89 & 18.42 & $17.18^{\mathrm{b}}$ & 18.99 & 6.00 & 22.58 \\
\hline
\end{tabular}

Data are represented as the differences from baseline in stratified subjects.

${ }^{\mathrm{a}}$ Means denote significance between placebo and treatment by Wilcoxon rank sum test; $P<0.05$.

${ }^{\mathrm{b}}$ Means denote significant increase after treatment by paired sample $t$-test; $P<0.05$.

LGNC-07 showed memory improvement by marginally increasing the delayed recognition of Rey-Kim memory test $(P=0.0572)$ and especially it significantly increases Rey-Kim MQ in stratified subjects (LGNC-07, $n$ 11; placebo, $n$ 9) whose MMSE-K lies between 21 and $23(P<0.05)$. Selective attention was also improved in the stratified subjects bya significant increase of word reading $(P<0.05)$. Therefore, this study suggests that LGNC-07 might be a potential nutraceutical candidate for cognition improvement.

1. Kim TI, Yuk DY, Park SG et al. (2008) Lab Anim Res 24, 87-92.

2. Petersen RC, Doody R, Kurz A et al. (2001) Arch Neurol 58, 1985-1992. 